\title{
Antibiotic resistance, serogroups, virulence genes, and phylogenetic groups of Escherichia coli isolated from yaks with diarrhea in Qinghai Plateau, China
}

\author{
Mujeeb Ur Rehman ${ }^{1}$, Hui Zhang ${ }^{1}$, Muhammad Kashif Iqbal', Khalid Mehmood ${ }^{1,3}$, Shucheng Huang ${ }^{1}$, \\ Fazul Nabi ${ }^{1}$, Houqiang Luo ${ }^{1}$, Yanfang Lan ${ }^{1}$ and Jiakui Li $i^{1,2^{*}}$
}

\begin{abstract}
Background: Ruminants serve as one of the most important reservoirs for pathogenic Escherichia coli. Infection with E. coli, a foodborne enteropathogen, can lead to asymptomatic infections that can cause life-threatening complications in humans. Therefore, from a clinical and human health perspective, it is important to know which virulence genes, phylogenetic groups, serogroups, and antibiotic resistance patterns are present in E. coli strains in yaks with diarrheic infections.

Methods: Two-hundred and ninety-two rectal swabs were collected from diarrheic yaks in Qinghai Plateau, China. The antimicrobial sensitivity of each resulting isolate was evaluated according to the disk diffusion method, and different PCR assays were performed for the detection of virulence genes and different phylogroups. Additionally, strains were allocated to different serogroups based on the presence of $O$ antigen via the slide agglutination method.

Results: Among the E. coli isolates tested, most of the isolates were multidrug resistant (97\%) and harbored at least one virulence gene (100\%). We observed ten virulence genes (sfa, eaeA, cnf1, etrA, papC, hlyA, aer, faeG, rfc, and sepA), of which sfa was the most commonly found (96.9\%). Significant positive associations between some resistance phenotypes and virulence genes were observed $(P<0.05, \mathrm{OR}>1)$. The majority of the $E$. coli isolates belonged to phylogroup A (79.5\%), and the others belonged to phylogroups B1 (7.5\%), D (4.1\%), B2 (5.8\%), and F (0.7\%). Among all the $E$. coli strains tested, serogroups $\mathrm{O}_{91}$ and $\mathrm{O}_{145}$ were the most prevalent, accounting for 15.4 and $14.4 \%$, respectively.

Conclusions: Our results suggest that yaks with diarrhea serve as a reservoir of pathogenic E. coli carrying various virulence genes and resistance phenotypes. Therefore, clinicians and relevant authorities must ensure the regulatory use of antimicrobial agents and prevent the spread of these organisms through manure to farm workers and foodprocessing plants.
\end{abstract}

Keywords: Antimicrobial sensitivity, Escherichia coli, Phylogeny, Serogroup, Virulence

\section{Background}

While Escherichia coli is an important part of the microbiota of the intestinal tract of animals and humans, certain $E$. coli pathotypes are implicated in different animal

\footnotetext{
*Correspondence: lijk210@sina.com

${ }^{1}$ College of Veterinary Medicine, Huazhong Agricultural University, Wuhan 430070, People's Republic of China

Full list of author information is available at the end of the article
}

and human infections $[1,2]$. The pathogenicity of E. coli is determined by particular virulence traits such as capsules, toxins, invasions, adhesions, haemolysins, cytotoxic necrotic factors, and effacement factors [3]. These pathogenic E. coli are classified into intestinal (InPEC) and extraintestinal pathogenic E. coli (ExPEC) based on the clinical signs and virulence factors [3]. The InPEC are mainly responsible for diarrheic infections and are the leading cause of mortality especially in children. On the 
other hand, ExPEC are responsible for infections outside the digestive tract such as urinary tract infections, meningitis, and septicemia $[1,3]$.

Domestic yaks are food animals, physiologically adapted to high altitude regions of southern central Asia, Mongolia, and Russia. However, frequent outbreaks of fatal hemorrhagic diarrhea in yaks are a serious concern from both a veterinary and a human health perspective $[2,4]$. As such, it is important to know, which virulence genes, phylogroups, serogroups, and antibiotic resistance patterns are present in commensal E. coli strains in yaks with diarrheic infections. Ruminants act as one of the most important reservoirs for pathogenic E. coli and lead to asymptomatic infections that can cause life-threatening complications in humans $[5,6]$. Therefore, this bacterium in yaks with diarrhea may be a potential health risk if it is transmitted to humans via cross-contamination of water, food, carcasses, or feces [7].

Bacterial infections are widely treated with a variety of antibiotics in both animals and humans [8]. However, misuse of antibiotics in clinical and veterinary settings has resulted in the emergence of multidrug-resistant microbes $[9,10]$. Researchers have characterized that antibiotic resistance is more common in pathogens compared to commensal organisms, and is linked to the association between resistance and virulence factors or due to frequent exposure of pathogenic strains to antibiotics [11]. However, reduced frequency of virulence determinants with high associations among resistance to certain antimicrobial agents is also reported in humans [12]. Therefore, these linkages are still not clear despite several studies. To the best of our knowledge, this is the first report examining the virulence genes, serogroups, phylogroups, and phenotypic resistance characteristics in E. coli strains isolated from yaks with diarrhea in China.

Currently, it is important to assess the risk of food animal-related (especially yaks) antibiotic resistance (AMR) and virulence factors on public health. Distribution of antibiotic resistance, serogroups, phylogroups, and associated virulence traits has not previously been observed in diseased or diarrheic yaks in China. This necessitates additional studies in such neglected food animals. Therefore, this study aims to characterize the possible association and distribution of phenotypes, virulence factors, phylogenetic groups, and serogroups of commensal $E$. coli strain isolated from yaks with diarrhea.

\section{Methods}

\section{Sample collection, isolation, and identification}

In this study, 292 rectal swabs were collected from adult yaks with diarrhea, in the Qinghai Plateau, China, between June 2015 and September 2016. Samples were collected from 37 different farms [Yushu, 20 farms $(n=173)$ and Haibei, 17 farms $(n=119)]$ from yaks raised for milk and meat purpose. Only one $E$. coli isolate was examined per rectal swab. Samples were transported to Huazhong Agricultural University (HZAU Wuhan, China) in ice-cooled containers for further experiments. All samples were enriched in nutrient broth and streaked on MacConkey agar (agar and broth media were purchased from GE Hangwei Medical Systems Co., Ltd., Beijing, China). Pink-colored colonies on MacConkey agar were subsequently used to inoculate eosin methylene blue agar (EMB), and greenish metallic-colored colonies on EMB were considered E. coli. Strains were then confirmed as E. coli via biochemical analysis, using the API 20E system (BioMerieux, Marcy-l Etoile, France; IMViC). Confirmed E. coli strains were suspended in Tryptic Soya Broth (TSB) and stored at $-80^{\circ} \mathrm{C}$ in $20 \%$ glycerol.

\section{Antibiotic sensitivity test}

The antibiotics used in this study were based on the information provided by farm veterinarians. The antimicrobial sensitivity profile of all $E$. coli isolates was determined using the disk diffusion method, according to the criteria described by the Clinical and Laboratory Standards Institute (CLSI) [13]. Mueller-Hinton agar was used as the test medium for each of the following antimicrobials (all purchased from GE Hangwei Medical Systems Co., Ltd., Beijing, China): ampicillin (AMP, $10 \mu \mathrm{g}$ ), ceftriaxone, (CEF, $30 \mu \mathrm{g})$, chloramphenicol (CHP, $30 \mu \mathrm{g}$ ), ciprofloxacin (CIP, $5 \mu \mathrm{g}$ ), gentamicin (GEN, $10 \mu \mathrm{g}$ ), streptomycin (STR, $10 \mu \mathrm{g})$, tetracycline (TET, $30 \mu \mathrm{g}$ ), and trimethoprim/sulfamethoxazole (SXT, 1.25/23.75 $\mu \mathrm{g}$ ). The test was done in triplicate for each strain and E. coli ATCC 25922 and Klebsiella pneumoniae ATCC 700603 was used as the positive and negative control strains, respectively.

\section{DNA extraction and screening for virulence-associated genes}

Total bacterial DNA was extracted from each strain via boiling, as previously described [14], and used as the DNA template in all PCRs. All samples were then subjected to uniplex or multiplex PCR assays with specific primers for the detection of virulence genes (all the virulence genes investigated in this study were chosen based on their functional characteristics (adhesions, toxins, and capsule synthesis) and association with E. coli InPEC or ExPEC pathotypes, as shown in Table 1) encoding est $A$, fasA, sepA [11], aer, cnf1 [15], eltA, exhA, faeG, hlyA, papC, $r f c, s f a$ [16], eaeA, stx1, stx2 [17], and etrA [18]. All testing was done with appropriate virulence genes as the positive control and sterile water as the negative control. Amplification reactions were carried out in 
Table 1 List of 16 virulence factors screened in present study, categorized based on their association with Escherichia coli pathotypes

\begin{tabular}{|c|c|c|c|c|}
\hline \multirow[t]{2}{*}{ Pathotype } & \multicolumn{3}{|c|}{ Virulence factor categories } & \multirow[t]{2}{*}{ Function } \\
\hline & Adhesins & Capsule synthesis & Toxins & \\
\hline \multirow[t]{6}{*}{ EXPEC } & sfa & & & S fimbriae (sialic acid-specific) \\
\hline & & & cnf1 & Cytotoxic necrotizing factor 1 \\
\hline & papC & & & Pilus associated with pyelonephritis (P fimbriae) \\
\hline & & & hlyA & a-Hemolysin \\
\hline & & $\mathrm{rfc}$ & & Lipopolysaccharide synthesis \\
\hline & $\operatorname{sep} A$ & & & Secreted serine protease of the auto-transporter family \\
\hline EAEC & etrA & & & Component of ETT2 type III secretion system \\
\hline EIEC & aer & & & Aerobatin \\
\hline \multirow[t]{4}{*}{ ETEC } & faeG & & & F4 fimbrial adhesion \\
\hline & fas $A^{b}$ & & & Fimbrial adhesion \\
\hline & & & elt $A^{b}$ & Heat-labile enterotoxin \\
\hline & & & est $A^{b}$ & Heat-stable enterotoxin \\
\hline \multirow[t]{2}{*}{ EPEC } & eae $A^{a}$ & & & Intimin/attaching and effacing \\
\hline & & & $e x h A^{a, b}$ & Enterohemolysin \\
\hline \multirow[t]{3}{*}{ EHEC } & eae $A^{a}$ & & & Intimin/attaching and effacing \\
\hline & & & $\begin{array}{l}s t \times 1^{b} \\
s t \times 2^{b}\end{array}$ & $\begin{array}{l}\text { Shiga-toxin-I } \\
\text { Shiga-toxin-II }\end{array}$ \\
\hline & & & $\operatorname{exh} A^{a, b}$ & Enterohemolysin \\
\hline
\end{tabular}

\footnotetext{
a Indicates genes shared by more than one $E$. coli pathotype
}

b Indicates none of the isolates were positive for these genes

$25 \mu \mathrm{L}$ volumes comprising $5 \mu \mathrm{L}$ genomic DNA, $12.5 \mu \mathrm{L}$ $2 \times$ reaction buffer, $1 \mu \mathrm{L}$ of each primer, $0.2 \mu \mathrm{L}$ gold DNA polymerase, and $5.3 \mu \mathrm{L} d \mathrm{ddH}_{2} \mathrm{O}$ using a Veriti thermal cycler (Applied Biosystems, Waltham, MA, USA). The thermocycler conditions were as follows: denaturation at $95{ }^{\circ} \mathrm{C}$ for 4 min followed by 25 cycles of denaturation at $94{ }^{\circ} \mathrm{C}$ for $30 \mathrm{~s}$, variable annealing for $30 \mathrm{~s}$, and extension at $72{ }^{\circ} \mathrm{C}$ for $30 \mathrm{~s}$.

\section{Phylogenetic analysis of $E$. coli strains}

Escherichia coli strains were assigned to phylogenetic groups A, B1, B2, C, D, E, or F by quadruplex PCR analysis using a previously described protocol by targeting chuA, yjaA, $\operatorname{arp} A$, and DNA fragment TspE4.C2 [19]. All testing was done with appropriate positive and negative controls. The results were interpreted as previously described [19].

\section{Serogrouping}

O sero-typing was carried out using all commercially available antisera based on the slide agglutination test as per the manufacturer's instructions (Tianjin Biochip co., LTD, Tianjin, China). For each test, polyvalent antisera and $0.5 \%$ phenol saline were also mixed together as a quality control.

\section{Statistical analysis}

Variables were expressed as percentages (\%). The significant association between antibiotic-resistant phenotypes, virulence genes, and phylogenetic groups were determined using the Pearson's Chi-squared test. $P$ values $<0.05$ were considered significant. Odds ratios (OR) and 95\% confidence intervals were determined, and $\mathrm{OR}<1$ and $>1$ represent negative and positive associations, respectively. All analyses were conducted using the Stata 11 software (StataCorp Lp, College Station, TX, USA).

\section{Results}

\section{Phenotypic antibiotic resistance}

The highest rates of resistance were to ampicillin (95.5\%), tetracycline $(90.1 \%)$, and gentamicin $(79.4 \%)$, with moderate rates of resistance to chloramphenicol (75.7\%) and ceftriaxone (72.6\%). However, 61.6, 56.8, and $43.8 \%$ of strains were resistant to trimethoprim/sulfamethoxazole, streptomycin, and ciprofloxacin, respectively (Table 2). Ninety-seven percent were MDR (resistant to at least three different classes of antibiotics), whereas $18 \%$ were resistant to all antimicrobials tested. Thirty-eight resistance patterns were observed, of which AMP-TETGEN-CHP-CEF-SXT-STR-CIP (17.8\%) was the most common. For statistical analysis, isolates of intermediate 
Table 2 Distribution of resistant phenotypes in different phylogroups detected in Escherichia coli strains isolated from yaks with diarrhea $(n=292)$

\begin{tabular}{|c|c|c|c|c|c|c|c|c|}
\hline \multirow{2}{*}{$\begin{array}{l}\text { Phylogroups } \\
\text { (no.) }\end{array}$} & \multicolumn{8}{|c|}{ Resistant phenotypes, no. } \\
\hline & $\operatorname{AMP}(n=279)$ & $\operatorname{TET}(n=263)$ & GEN $(n=232)$ & $\mathrm{CHP}(n=221)$ & CEF $(n=212)$ & $\operatorname{SXT}(n=180)$ & $\operatorname{STR}(n=166)$ & $\mathrm{CIP}(n=128)$ \\
\hline $\mathrm{A}(n=232)$ & 223 & 204 & 195 & 189 & 166 & 138 & 132 & 99 \\
\hline $\mathrm{B} 1(n=22)$ & 22 & 22 & 17 & 6 & 17 & 18 & 14 & 11 \\
\hline $\mathrm{B} 2(n=12)$ & 12 & 12 & 3 & 11 & 10 & 9 & 7 & 0 \\
\hline $\mathrm{D}(n=17)$ & 17 & 17 & 14 & 14 & 15 & 13 & 13 & 9 \\
\hline $\mathrm{F}(n=2)$ & 2 & 2 & 1 & 0 & 2 & 2 & 0 & 2 \\
\hline $\mathrm{ND}(n=7)$ & 3 & 6 & 2 & 1 & 2 & 0 & 0 & 7 \\
\hline
\end{tabular}

AMP, ampicillin; TET, tetracycline; GEN, gentamicin; CHP, chloramphenicol; CEF, ceftriaxone; SXT, trimethoprim/sulfamethoxazole; STR, streptomycin; CIP, ciprofloxacin; ND, strains that were not assigned to any phylogroup

susceptibility were considered to be sensitive. Table 2 summarizes the distribution of resistance phenotypes in different phylogroups detected in E. coli strains isolated from yaks with diarrhea.

\section{Prevalence of virulence genes}

Ten out of 16 virulence genes were detected, namely, sfa (96.9\%), eaeA (68.2\%), cnf1 (46.2\%), etrA (24.3\%), papC (23.3\%), hlyA (17.5\%), aer (12.7\%), faeG (4.8\%), rfc (1.7\%), and sep $A(0.7 \%)$. However, none of the isolates were positive to stx1, stx2, exhA, eltA, est $A$, and fas A. Furthermore, the $s f a$ gene was most prevalent (26.7\%) among the 22 virulence gene profile observed. Table 3 summarizes the distribution of virulence genes in different phylogroups and serogroups detected in E. coli strains isolated from yaks with diarrhea.

\section{Occurrence of $E$. coli pathotypes}

To identify the occurrence of InPEC and ExPEC pathotypes of $E$. coli, virulence genes were grouped according to their association with different pathotypes. Overall, majority of the E. coli isolates carried the combinations of virulence genes, associated with both intestinal and extraintestinal pathotypes (71.2\%). In addition, 27.1 and $1.7 \%$ of the isolates were positive for virulence genes associated with ExPEC and InPEC pathotypes, respectively. In the present study, all the tested virulence genes associated with ExPEC pathotype (100\%) were positive for at least two isolates. Conversely, $60 \%$ of the virulence genes associated with InPEC pathotypes were not positive for a single isolate (indicated in the Table 1).

\section{Occurrence of phylogroups}

The majority of the E. coli isolates belonged to phylogroup A (79.5\%), with the other isolates belonging to phylogroup B1 $(7.5 \%), \mathrm{B} 2(4.1 \%), \mathrm{D}(5.8 \%)$, and $\mathrm{F}$ (0.7\%). However, 7 isolates (2.4\%) were not assigned to any phylogenetic group. Tables 2 and 3 summarize the distributions of antibiotic resistance phenotypes, serogroups, and virulence genes among the different phylogenetic groups examined. Due to the small number of strains identified in the other phylogroups, associations between antibiotic resistance phenotypes and virulence genes were analyzed only among strains of phylogroup A (Tables 4 and 5). There was a varied statistically significant association $(P<0.05)$ between the presence of the different antimicrobial resistance phenotypes and virulence genes. Overall, a negative association was more prevalent than a positive one but the strongest positive association was detected between cnf1/ampicillin and $\operatorname{sep} A / r f c$ gene pairs.

\section{Occurrence of serogroups}

The distribution of serotypes varied in E. coli strains isolated from yaks with diarrhea. Of the E. coli strains tested, serogroups $\mathrm{O}_{91}$ and $\mathrm{O}_{145}$ were the most prevalent, accounting for 15.4 and $14.4 \%$, respectively. Lastly, $8.9 \%$ of the isolates were untyped with the available antisera as shown in Table 2.

\section{Discussion}

In present study, we observed a higher proportion of multidrug-resistant $E$. coli with virulence factors in yaks suffering from diarrheic infections, and determined the correlations among virulence genes and resistance phenotypes. These data can be compared to the reports of other regions and in other animals since the study of antimicrobial resistance in important food animals such as yak is still inadequate.

In this study, ninety-seven percent of the E. coli isolates were resistant to at least three different classes of antibiotics (MDR), whereas $18 \%$ were resistant to all antimicrobials tested. The phenotypic resistance to ampicillin and tetracycline was identified at a high rate, similar to the previous findings in the isolates from diarrheic or diseased animals in China [17, 20-23]. The predominance 


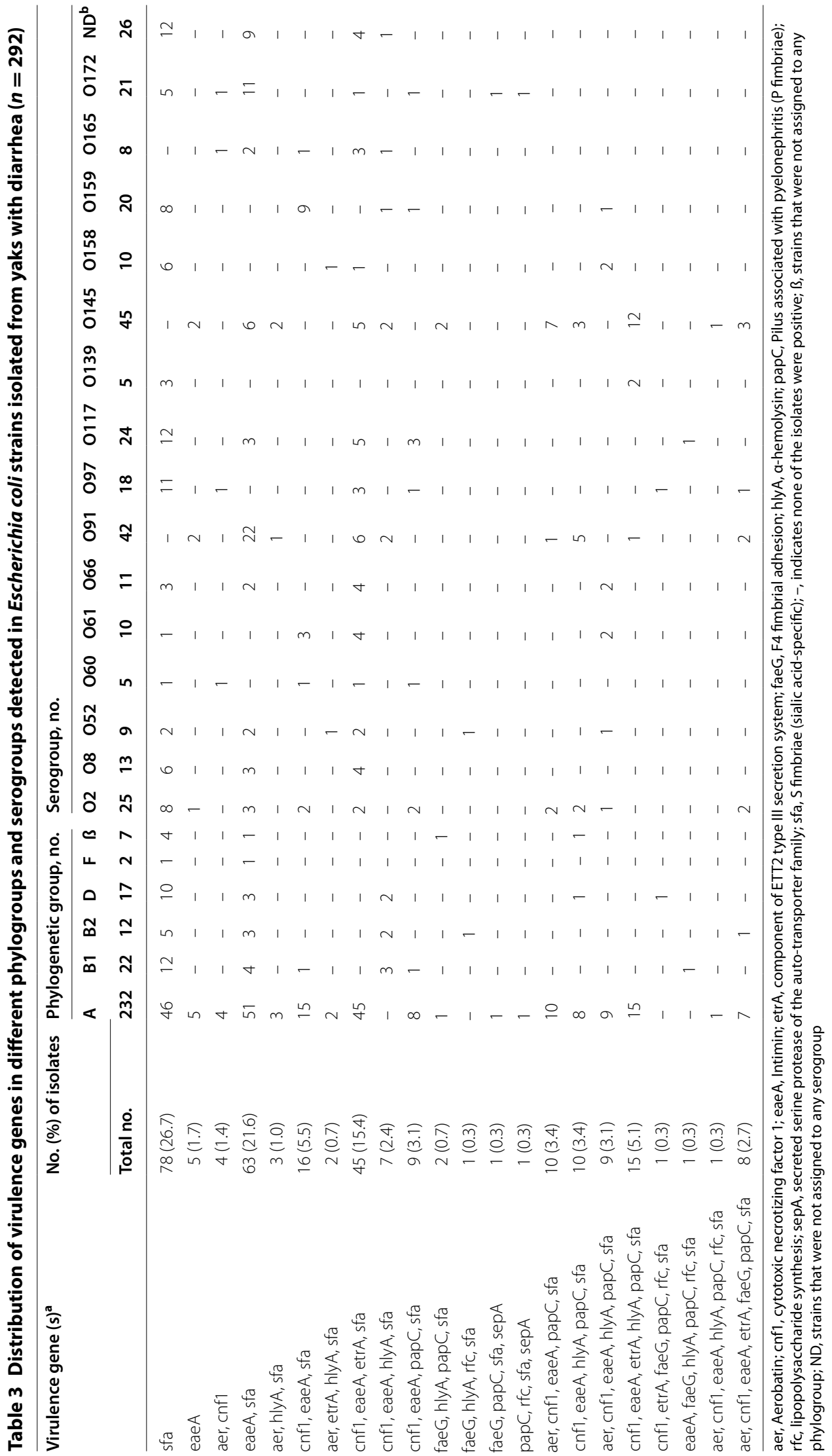




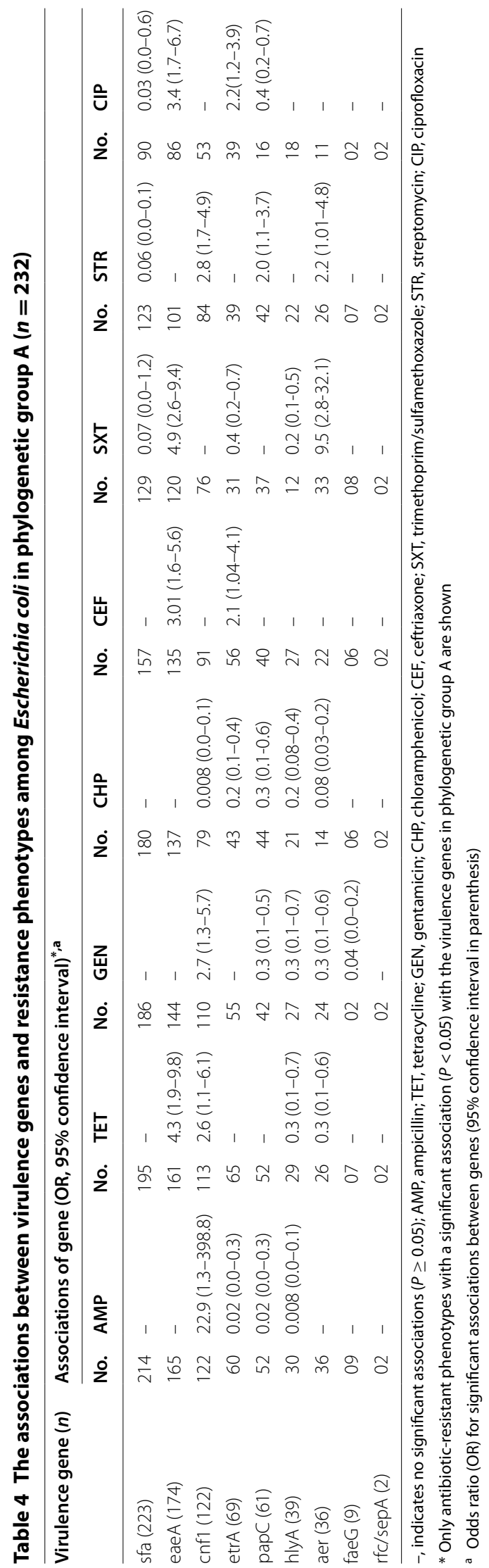




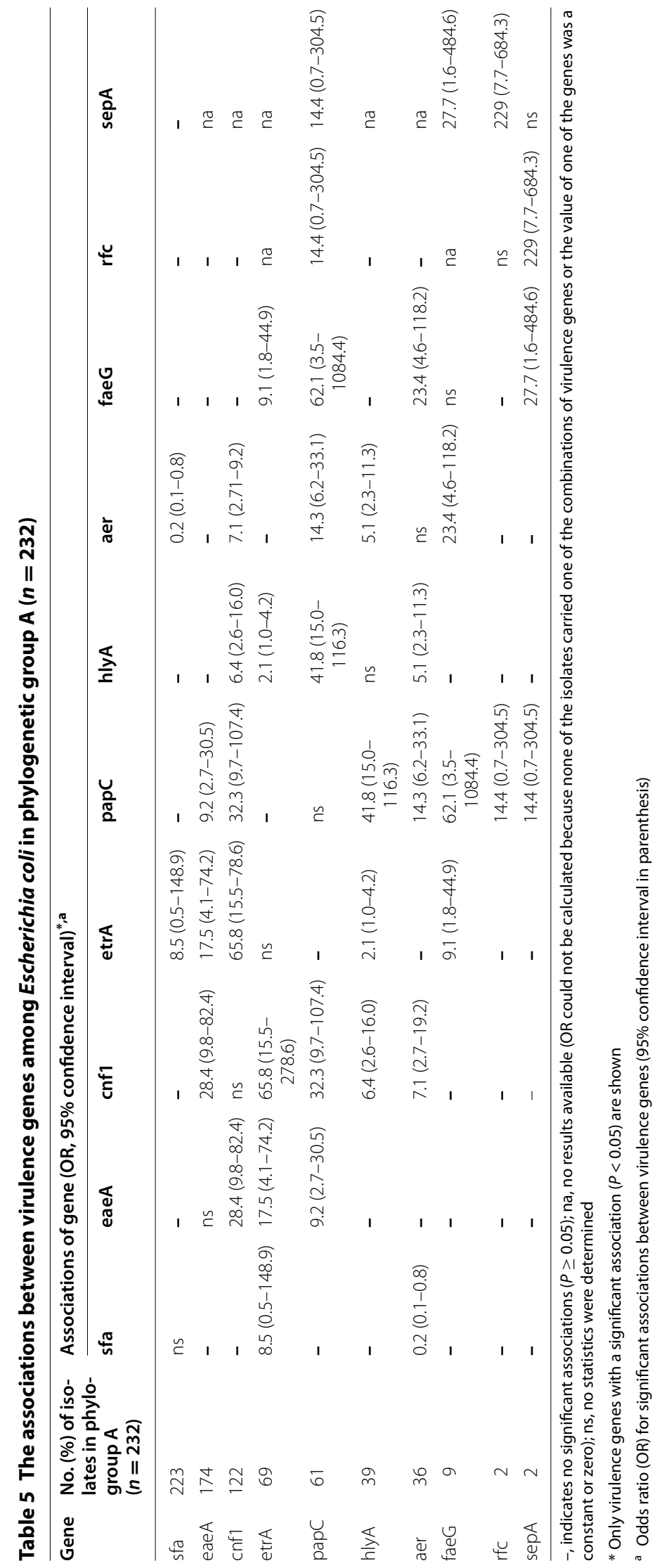


of tetracycline resistance among the E. coli strains from diarrheic yaks was similar to the findings of Boerlin et al. [11], who detected tetracycline resistance in 96 out of 100 E. coli strains isolated from diarrheic pigs. However, similar levels of resistance were observed in $E$. coli strains isolated from healthy pigs and chickens [24]. Altogether, these findings reflect the widespread and heavy use of such antibiotics in animals in China. Approximately $30 \%$ of drugs sold in China are antibiotics, which is $20 \%$ higher than the proportion in the developed world [25]. Furthermore, China has the highest rate of antimicrobial resistance (enteric gram-negative bacilli) in both community and hospital-acquired infections among Asian courtiers, along with Singapore and Philippines [26]. As such, antimicrobial resistance is a major public health concern in China [27]. The use of chloramphenicol in food animals is banned, as there is a high frequency of chloramphenicol-resistant phenotypes. The high levels of chloramphenicol resistance have been formerly reported in other bacteria of animal origin and are probably linked to the proficient horizontal dissemination of resistance determinants or co-selection of resistant genes [28]. Furthermore, the high resistance to antibiotics in the study area may be a sign of difference in disease control practices, antimicrobial usage, or various unknown factors such as genetic mutations contributing towards multidrug-resistant phenotypes [29]. Therefore, strong surveillance programs are needed to control the dissemination of antibiotic resistance in nomadic pastorals of China like Qinghai-Tibetan Plateau.

In present study, various virulence genes were identified in the E. coli isolates, suggesting the existence of pathogenic E. coli in yaks with diarrhea. Overall, our results showed that a significant fraction of $E$. coli isolates from diarrheic yaks are possible diarrheagenic and extraintestinal pathotypes. It is particularly worrisome that all $(100 \%)$ the tested virulence genes, associated with ExPEC pathotype (100\%) were positive for at least two isolates. Conversely, $60 \%$ of the InPEC pathotype did not reveal any associated virulence gene under investigation. This observation indicates a high potential health concern as virulence genes associated with ExPEC pathotype were more common in diarrheic yaks, which is considered to be a possible health risk due to their pathogenic potential [3]. Moreover, our results present a possibility that the observed combinations of virulence genes are involved in a distinct category of diarrheagenic E. coli. Nevertheless, the occurrence of single or multiple virulence factors in an E. coli strain does not essentially signify that a strain is pathogenic because $E$. coli uses a multifaceted mechanism of pathogenesis [30, 31]. Therefore, further studies in animal model or tissue culture are required to demonstrate the pathogenicity of observed virulence genes/patterns.

In addition, we observed a moderate number of virulence-associated genes of both InPEC and ExPEC categories in diarrheic yaks. This could be explained by the harsh environmental conditions at Qinghai-Tibetan Plateau (average altitude $3000 \mathrm{~m}$ ). Yaks are physiologically adapted to high altitude environmental conditions (hypoxia, $\mathrm{pH}$, and high altitude radiations) that genetically equip them with relatively stronger ability of resistance or tolerance to infections [32,33]. In present study, $s f a$ and $e a e A$ were the most abundant virulence genes, which are linked with ExPEC and InPEC pathotypes, respectively. Conversely, all isolates were negative for the InPEC-associated toxin genes (stx1, stx2, exhA, eltA, and est $A$ ), but positive for ExPEC-associated toxin genes (cnf1 and hlyA). Such observation has not been commonly described in previous reports of $E$. coli isolates of animal origin $[2,7,10,17]$. This observed variation could be attributed to the existing climatic conditions which may account for the diverse occurrence of virulenceassociated genes. Furthermore, we also observed that a relatively high number of $E$. coli isolates carried a combination of $s f a$ and eaeA genes. The exact significance of this combination is not clear. However, the eaeA gene is involved in adherence to epithelial cells [producing characteristic attaching and effacing (A/E) lesions] [34] and $s f a$ may possibly assist with the fixation of $E$. coli in the gastrointestinal tract of yaks. Further studies are required to understand this phenomenon. In addition, the occurrence of unusual patterns of virulence genes observed in current study might be due to horizontal gene transfer (integrons, plasmids, transposons) between related or unrelated bacterial species [35].

The association among virulence genes and resistance phenotypes varied in this study. Overall, negative correlations were more common between virulence genes and phenotypic resistance. We observed the strongest association between ExPEC-associated toxin gene (cnf1) and ampicillin. In addition, EHEC-associated adhesion gene $(e a e A)$ was the most prevalent associated gene with the resistance to tetracycline, ceftriaxone, ciprofloxacin, and trimethoprim/sulfamethoxazole. Aerobain (aer) and component of ETT2 type III secretion system (etrA) were also significantly associated with the resistance to ceftriaxone, ciprofloxacin, and streptomycin, trimethoprim/ sulfamethoxazole, respectively. Furthermore, resistance to streptomycin was significantly associated with increased frequency of papC, and cnfl. Such associations were not observed in previous studies $[10,15,21]$. Our findings suggest that the association of virulence and resistance might be strain-specific or due to various 
antibiotics used in different geographical regions. Notably, negative correlations were not observed among virulence genes (except aer and sfa gene pairs), and strongest associations were observed among virulence genes, sep $A$ and $r f c$ gene pairs followed by etA/cnf1 and faeG/papC gene pairs. Such associations were not observed in previous studies $[10,15,21]$. Taken together, these findings suggest that associations among resistance and virulence genes in $E$. coli isolates vary with antimicrobial use and to a certain phylogenetic background. Moreover, we examined only the phenotypic profile of our isolates (in vitro). Therefore, further studies are required to elaborate the real significance of the observed associations and its impact on different outcomes of infection.

All the E. coli strains were allocated to phylogenetic groups, A, B1, B2, C, D, E, and F as previously suggested [19]. Based on the phylogenetic analysis, antibiotic-resistant $E$. coli isolates of animal origin were not associated with low virulence traits [12, 36]. It has been reported that ExPEC, a prominent zoonotic infection that is responsible for urinary tract infections in humans, is mainly associated with groups B2 and D [37]. Especially, extraintestinal virulence is considered to be epidemiologically linked with the phylogroup B2 by means other than the known extraintestinal virulence factors [38]. In contrast, groups A and B1 were reported to be associated with InPEC and commensal strains [39]. In this study, the majority of the E. coli isolates belonged to group A $(79.5 \%)$ and the remaining to phylogroups B1, B2, D, or F. Moreover, E. coli adhesion gene (sfa) was the most common gene observed in phylogroup A strains. The exact significance of this combination is not clear. However, it suggests a possible role of this combination (phylogroup A and adhesion gene, $s f a$ ) in the diarrheic infections, as these strains were obtained from yaks with diarrhea. Furthermore, the high prevalence of phylogroup A identified was consistent with the appurtenance of the isolates examined in this study, and our findings were in line to those noted in some previous studies of diarrheagenic and commensal strains $[40,41]$.

We observed 15 different $\mathrm{O}$ serotypes among the diarrheic yaks. Interestingly, we found that O91 and O145 had the highest frequency of virulence genes. STEC serogroup O157 and non-O157 strains such as O26, O91, O103, O111, O113, O128, O121, and O145 have been shown to cause diarrhea $[42,43]$. The high prevalence of O91 and O145 identified was inconsistent with the findings of previous reports from patients with diarrhea $[44,45]$. Serogroups O2, O8, O60, O61, O66, O91, O97, O117, O158, O159, O165, and O172 were earlier identified in humans and animals with diarrhea $[2,6,46,47]$. However, the serogroups $\mathrm{O} 2$ and $\mathrm{O} 60$ were also reported in MDR uropathogenic isolates of $E$. coli from patients with prostatitis, simple UTI, pyelonephritis, and cystitis in India [29]. Lastly, the serogroups O52 and O139 detected in this study appear to be additional serotypes associated with diarrhea in yaks. If these yaks do not receive effective treatment, they are prone to secondary infections and diseases.

\section{Conclusion}

The findings of the present study highlight the important role of yaks as a potential reservoir of drug-resistant $E$. coli with a variety of virulent determinants that may spread into the environment and to humans. The association between resistance and virulence genes sustains the concerns that virulence traits in yaks can be selected by antibiotic usage in the farms. Therefore, we recommend that strong surveillance programs be initiated to control and monitor the frequency and regulatory use of antimicrobial agents.

\section{Authors' contributions}

$J L$ and MUR participated in the conception and design of the study and wrote the manuscript. MUR and $\mathrm{HZ}$ performed the experiments. MKI, KM, SH, $\mathrm{FN}, \mathrm{HL}$, and $\mathrm{YL}$ analyzed the data. All authors contributed to the analysis and supported the manuscript discussion. All authors read and approved the final manuscript.

\section{Author details \\ ${ }^{1}$ College of Veterinary Medicine, Huazhong Agricultural University, Wuhan 430070, People's Republic of China. ${ }^{2}$ Laboratory of Detection and Monitoring of Highland Animal Diseases, Tibet Agricultural and Animal Husbandry College, Linzhi 860000, Tibet, People's Republic of China. ${ }^{3}$ Univer- sity College of Veterinary and Animal Sciences, Islamia University of Bahawal- pur, Bahawalpur 63100, Punjab, Pakistan.}

\section{Acknowledgements}

MUR acknowledges China Scholarship Council (CSC) for providing fellowship. We would also like to sincerely thank the anonymous reviewers for very accurate recommendations and comments that helped us to improve the overall quality of this article.

\section{Competing interests}

The authors declare that they have no competing interests.

\section{Availability of data and materials}

The data supporting the findings of this study are contained within the manuscript.

\section{Ethics approval and consent for participate}

Permission for specific locations, information regarding the number of samples harvested, and an associated permit number for yaks were not required, and no endangered or protected species were involved or harmed during this study.

\section{Funding}

This study was supported by the Tibet Autonomous Region Science Fund and the Chinese Agricultural Research Systems (CARS-38); Agriculture and Animal Husbandry Research Collaborative Innovation Project: yak important epidemiological disease investigation and prevention research by the Agricultural and Animal Husbandry College of Tibet University.

\section{Publisher's Note}

Springer Nature remains neutral with regard to jurisdictional claims in published maps and institutional affiliations. 
Received: 24 February 2017 Accepted: 21 April 2017 Published online: 28 April 2017

\section{References}

1. Nataro JP, Kaper JB. Diarrheagenic Escherichia coli. Clin Microbiol Rev. 1998;11:142-201.

2. Bandyopadhyay S, Lodh C, Sarkar M, Ghosh MK, Bera AK, Bhattacharyya $D$, et al. Prevalence molecular fingerprinting and drug resistance profile of enterovirulent Escherichia coli isolates from free ranging yaks of Tawang district, Arunachal Pradesh, India. Trop Anim Health Prod. 2012:44:1063-72

3. Kaper JB, Nataro JP, Mobley HL. Pathogenic Escherichia coli. Nat Rev Microbiol. 2004;2:123-40.

4. CergoleNovella MC, Nishimura LS, Dos Santos LF, Irino K, Vaz TM, Bergamini AM, et al. Distribution of virulence profiles related to new toxins and putative adhesins in Shiga toxin producing Escherichia coli isolated from diverse sources in Brazil. FEMS Microbiol Lett. 2007:274:329-34.

5. Wani SA, Hussain I, Nabi A, Fayaz I, Nishikawa Y. Variants of eae and stx genes of atypical enteropathogenic Escherichia coli and nonO157 Shiga toxin-producing Escherichia coli from calves. Lett App Microbiol. 2007:45:610-5.

6. Kim YJ, Kim JH, Hur J, Lee JH. Isolation of Escherichia coli from piglets in South Korea with diarrhea and characteristics of the virulence genes. Can J Vet Res. 2010;74:59-64.

7. Diarrassouba F, Diarra MS, Bach S, Delaquis P, Pritchard J, Topp E, et al. Antibiotic resistance and virulence genes in commensal Escherichia coli and Salmonella isolates from commercial broiler chicken farms. J Food Prot. 2007:70:1316-27.

8. Erb A, Stürmer T, Marre R, Brenner H. Prevalence of antibiotic resistance in Escherichia coli: overview of geographical, temporal, and methodological variations. Eur J Clin Microbial Infect Dis. 2007;26:83-90

9. Wang XM, Liao XP, Liu SG, Zhang WJ, Jiang HX, Zhang MJ, et al. Serotypes, virulence genes, and antimicrobial susceptibility of Escherichia coli isolates from pigs. Foodborne Pathog Dis. 2011:8:687-92.

10. Schierack P, Steinruck H, Kleta S, Vahjen W. Virulence factor gene profiles of Escherichia coli isolates from clinically healthy pigs. Appl Environ Microbiol. 2006;72:6680-6.

11. Boerlin P, Travis R, Gyles CL, Reid-Smith R, Janecko N, Lim H, et al. Antimicrobial resistance and virulence genes of Escherichia coli isolates from swine in Ontario. Appl Environ Microbiol. 2005;71:6753-61.

12. Johnson JR, vander Schee C, Kuskowski MA, Goessens W, van Belkum A. Phylogenetic background and virulence profiles of fluoroquinoloneresistant clinical Escherichia coli isolates from the Netherlands. J Infect Dis. 2002;186:1852-6.

13. Clinical and Laboratory Standards Institute (CLSI). Performance standards for antimicrobial susceptibility testing. Twenty-First Informational Supplement. CLSI/NCCLS-M100-S24. Wayne: Clinical and Laboratory Standards Institute; 2014.

14. Levesque $C$, Piche L, Larose $C$, Roy PH. PCR mapping of integrons reveals several novel combinations of resistance genes. Antimicrob Agents Chemother. 1995;39:185-91

15. Alabsi MS, Ghazal A, Sabry SA, Alasaly MM. Association of some virulence genes with antibiotic resistance among uropathogenic Escherichia coli isolated from urinary tract infection patients in Alexandria, Egypt: a hospital-based study. J Global Antimicrob Res. 2014;2:83-6.

16. Chapman TA, Wu XY, Barchia I, Bettelheim KA, Driesen S, Trott D, et al. Comparison of virulence gene profiles of Escherichia coli strains isolated from healthy and diarrheic swine. Appl Environ Microbiol. 2006;72:4782-95

17. Cheng D, Sun H, Xu J, Gao S. PCR detection of virulence factors genes in Escherichia coli isolates from weaned piglets with edema disease and/or diarrhea in China. J Vet Microbiol. 2006;115:320-8.

18. Hartleib S, Prager R, Hedenstrom I, Lofdahl S, Tschape H. Prevalence of the new, SPI1-like, pathogenicity island ETT2 among Escherichia coli. Int J Med Microbiol. 2003:292:487-93.

19. Clermont O, Christenson JK, Denamur E, Gordon DM. The Clermont Escherichia coli phylo-typing method revisited: improvement of specificity and detection of new phylo-groups. Environ Microbiol Rep. 2013;5:58-65.

20. Chen X, Gao S, Jiao X, Liu XF. Prevalence of serogroups and virulence factors of Escherichia coli strains isolated from pigs with postweaning diarrhoea in eastern China. Vet Microbiol. 2004;103:13-20.

21. Jiang HX, Lu DH, Chen ZL, Wand XM, Chen JR, Liu YH, et al. High prevalence and widespread distribution of multi-resistant Escherichia coli isolates in pigs and poultry in China. Vet J. 2011;187:99-103.

22. Zhang $H$, Rehman MU, Li K, Luo H, Lan Y, Nabi F, et al. Antimicrobial resistance of Escherichia coli isolated from Tibetan piglets suffering from white score diarrhea. Pak Vet J. 2017:37:43-6.

23. Yang H, Chen S, White DG, Zhao S, McDermott P, Meng J. Characterization of multiple-antimicrobial-resistant Escherichia coli isolates from diseased chickens and swine in China. J Clin Microbiol. 2004;42:3483-9.

24. ChunPing Z, YiBao N, Song L. Resistance to tetracycline and distribution of tetracycline resistance determinants in commensal Escherichia coli isolated from clinically healthy chickens and pigs. Sci Agri Sin. 2010;12:2578-83.

25. Jia HP. Antibiotic resistance and the developing world. Science and Development Network; 2008. http://www.scidev.net/global/health/feature/antibiotic-resistance-and-the-developing-world.html. Accessed 22 Feb 2017).

26. Hirakata Y, Matsuda J, Miyazaki Y, Kamihira S, Kawakami S, Miyazawa $Y$, et al. Regional variation in the prevalence of extended-spectrum $\beta$-lactamase-producing clinical isolates in the Asia-Pacific region (SENTRY 1998-2002). Diagn Microbiol Infect Dis. 2005;52:323-9.

27. Xiao Y, Wang J, Li Y. MOH (National Antimicrobial Resistance Investigation Net). Bacterial resistance surveillance in China: a report from Mohnarin 2004-2005. Eur J Clin Microbiol Infect Dis. 2008:27:697-708.

28. Bischoff KM, White DG, Hume ME, Poole TL, Nisbet DJ. The chloramphenicol resistance gene $\mathrm{cm} / \mathrm{A}$ is disseminated on transferable plasmids that confer multiple-drug resistance in swine Escherichia coli. FEMS Microbiol Lett. 2005:243:285-91.

29. Jadhav S, Hussain A, Devi S, Kumar A, Parveen S, Gandham N, et al. Virulence characteristics and genetic affinities of multiple drug resistant uropathogenic Escherichia coli from a semi urban locality in India. PLoS ONE. 2011;6(3):e18063.

30. Huang DB, Mohanty A, DuPont HL, Okhuysen PC, Chiang T. A review of an emerging enteric pathogen: enteroaggregative Escherichia coli. J Med Microbiol. 2006:55:1303-11.

31. Gassama-Sow A, Sow PS, Gueye M, Gueye-N diaye A, Perret JL, Mboup S, et al. Characterisation of pathogenic Escherichia coli in human immunodeficiency virus-related diarrhoea in Senegal. J Infect Dis. 2004;189:75-8.

32. Qiu Q, Zhang G, Ma T, Qian W, Wang J, Zhiqiang Y, et al. The yak genome and adaptation to life at high altitude. Nat Genet. 2012;44:946-9.

33. Mirkena T, Duguma G, Haile A, Tibbo M, Okeyo AM, Wurzinger M, et al. Genetics of adaptation in domestic farm animals: a review. Livest Sci. 2010;2010(132):1-12.

34. Wales AD, Woodward MJ, Pearson GR. Attaching-effacing bacteria in animals. J Comp Pathol. 2005;132:1-26.

35. Nolonwabo N, Sibanda T, Nqwenya E. Okoh Al. Prevalence and antibiogram profiling of Escherichia coli pathotypes isolated from the Kat River and the Fort Beaufort abstraction water. Int J Environ Res Public Health. 2014;11:8213-27

36. Carlos C, Pires MM, Stoppe NC, Hachich EM, Sato MI, Gomes TA, et al. Escherichia coli phylogenetic group determination and its application in the identification of the major animal source of fecal contamination. BMC Microbiol. 2010;10:161

37. Nandanwar N, Janssen T, Kuhl M, Ahmed N, Ewers C, Wieler LH. Extraintestinal pathogenic Escherichia coli (ExPEC) of human and avian origin belonging to sequence type complex 95 (STC95) portray indistinguishable virulence features. Int J Med Microbiol. 2014;304:835-42.

38. Hussain A, Ranjan A, Nandanwar N, Babbar A, Jadhav S, Ahmed N. Genotypic and phenotypic profiles of Escherichia coli isolates belonging to clinical sequence type 131 (ST131), clinical non-ST131, and fecal non-ST131 lineages from India. Antimicrob Agents Chemother. 2014;58:7240-9.

39. Dobrindt U. (Patho-) genomics of Escherichia coli. Int J Med Microbiol. 2005:295:357-71.

40. Usein CR, Tatu-Chitoiu D, Ciontea S, Condei M, Damian M. Escherichia coli pathotypes associated with diarrhea in Romanian children younger than 5 years of age. Jpn J Infect Dis. 2009;62:289-93. 
41. Clermont O, Bonacorsi S, Bingen E. Rapid and simple determination of the Escherichia coli phylogenetic group. Appl Environ Microb. 2000;66:4555-8.

42. Schmidt $H$, Beutin L, Karch H. Molecular analysis of the plasmid-encoded hemolysin of Escherichia coli O157:h7 strain EDL 933. Infect Immun. 1995;63:1055-61.

43. Kappeli U, Hächler H, Giezendanner N, Beutin L, Stephan R. Human infections with non-O157 Shiga toxin-producing Escherichia coli, Switzerland, 2000-2009. Emerg Infect Dis. 2011;17:180-5.

44. Johnson KE, Thorpe CM, Sears CL. The emerging clinical importance of non-O157 Shiga toxin-producing Escherichia coli. Clin Infect Dis. 2006:43:1587-95.
45. Aslani MM, Alikhani MY. Serotypes of Enteropathogenic Escherichia coli isolated from children under 5 years of age. Iran J Publ Health. 2009;38:70-7.

46. Bai X, Zhao A, Lan R, Xin Y, Xie H, Meng Q, et al. Shiga toxin producing Escherichia coli in yaks (Bos grunniens) from the Qinghai Tibetan Plateau, China. PloS ONE. 2013;8:e65537.

47. Momtaz H, Dehkordi FS, Hosseini MJ, Sarshar M, Heidari M. Serogroups, virulence genes and antibiotic resistance in Shiga toxin-producing Escherichia coli isolated from diarrheic and non-diarrheic pediatric patients in Iran. Gut Pathog. 2013;5:39.

\section{Submit your next manuscript to BioMed Central and we will help you at every step:}

- We accept pre-submission inquiries

- Our selector tool helps you to find the most relevant journal

- We provide round the clock customer support

- Convenient online submission

- Thorough peer review

- Inclusion in PubMed and all major indexing services

- Maximum visibility for your research

Submit your manuscript at www biomedcentral com/submit 